\title{
Efficient Building of Interactive Applications Guided by Requirements Models
}

\author{
Begoña Losada, Maite Urretavizcaya, and Isabel Fernández de Castro \\ Dpt. of Computer Languages and Systems, University of the Basque Country \\ 649 p.k.- 20018 Donostia-San Sebastián, Spain \\ \{b. losada, maite.urretavizcaya, isabel. fernandez\} @ehu.es
}

\begin{abstract}
The analysis of functional and non-functional requirements of an interactive application not only encompasses the descriptive aspects of its functionality and the user and system restrictions, it must also satisfy user needs, such as usability, along with system needs, such as reliability. InterMod is an interactive application-design methodology with agile characteristics which proposes the use of incremental models to define requirements, configure presentation and develop functionality. This poster is focused on the requirements of the InterMod methodology for the efficient construction of interactive applications. These models include user, system, task and dialog models. An example created with a tool that follows these principles and illustrates our approach to the dialog model is presented.
\end{abstract}

Keywords: Requirements analysis, methodology, user model, system model, task model, dialog model.

\section{Introduction}

Techniques for programming functionality have traditionally taken precedence over human-computer interaction techniques. Therefore, it is common for users to feel that their needed functions are difficult to understand or unnatural.

The requirements capture stage identifies the capabilities that the software system must have in order to meet user needs. In this phase, user participation is particularly active. Thus, a language mutually understood by all people involved should be used in order to allow accurate evaluation.

According to [1], user requirements and user interface design should drive the overall development process. In addition, we believe that requirement gathering and prototype stages help to organize and check information presented in the interface as well as to plan, guide and verify the development process. In order to do this, user characteristics and behaviour should be taken into account along with the physical limitations of the device and the system's reactions.

Below, we present a brief introduction to the InterMod methodology and the models of detailed requirements. After that we provide an example of a dialog model. Finally, we present our conclusions. 


\section{The Requirements Models in the Intermod Methodology}

The objective of the InterMod methodology [2] is to facilitate the design of quality interactive applications. InterMod follows the Object Management Group's Model Driven Architecture proposal [3]. We propose interactive software development based on user-centred models generated and evaluated during different phases. Two noteworthy characteristics of InterMod are (a) its use of an Intermediate Language Description, which allows information generated in different models to be stored, and (b) its iterative nature, much like that of an agile process [4].

InterMod models make it possible to quickly produce incremental prototypes by adapting the design according to the modifications prompted by both user and software developer evaluations.

Figure 1 shows a diagram of InterMod's model connections. The user interface models have been separated from the functionality models. Despite this, the InterMod process promotes the early integration of interface models with system functionality.

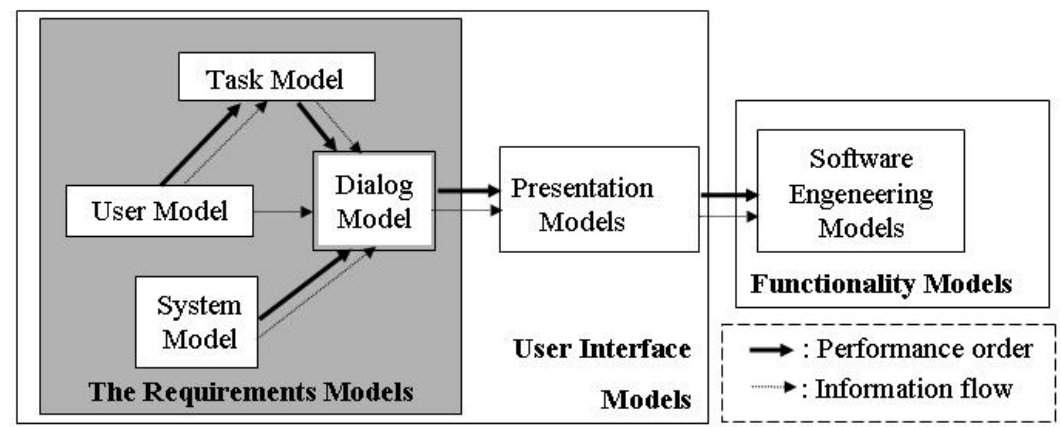

Fig. 1. InterMod models

When it comes to the requirements models, the user model, system model, task model and dialog model are all marked. First of all, the user model, which recognizes properties and limitations of the user, is created. The task model, which describes user performance in completing each task, follows the user model. The system model deals with the properties and limitations of the system. Finally, a dialog model is proposed using information from the other three requirements models.

The dialog model, known as the action-reaction model, represents user actions and system responses. The information in the dialog model affects the user interaction process, particularly the established order of navigation.

The software prototypes that are obtained from the models and tested at different times become the primary measure of progress. In addition, we suggest organizing and dividing the entire project by means of the dialog model, which should include the general overview of the navigation and the connections between user tasks and system actions.

Although UML use cases [5] are the usual means of capturing software system requirements, our proposed dialog model uses a task hierarchy that is not centred on establishing the programme's complete semantics. Our interest resides in identifying 
the critical aspects of navigation during user-system interactions. In this way, the interface and software analyses are facilitated through the use of early prototypes with user actions and the critical system responses that alter the normal flow of navigation.

\section{An example of a dialog model}

A dialog model created with a tool which implements InterMod specifications by means of XML descriptions can be seen in Figure 2. User actions are shown in square boxes while system reactions are represented by ovals.

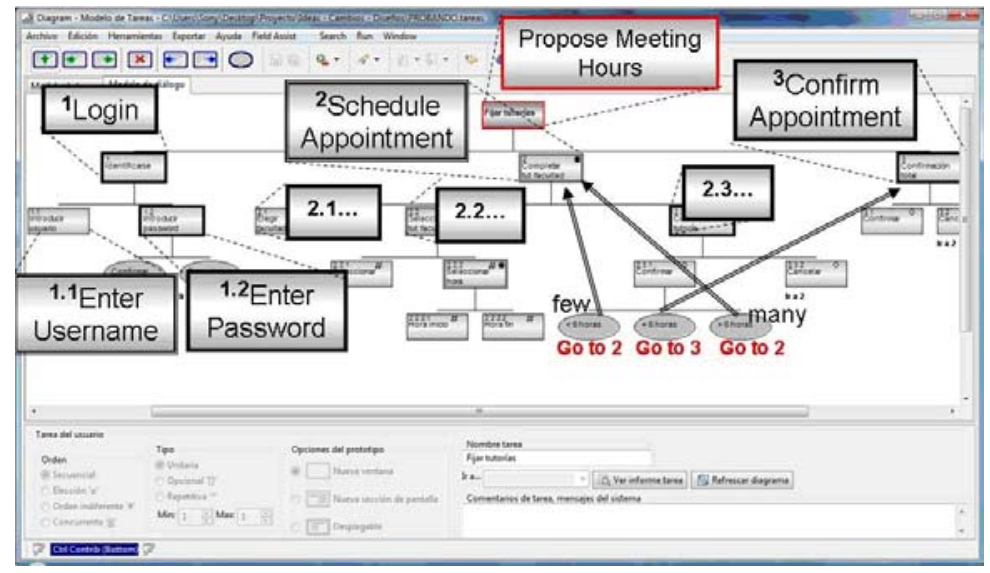

Fig. 2. An example of a dialog model

In this example, the task hierarchy of task "Propose Meeting Hours" is illustrated. The established subtask order is: (a) "1.Login", (b) "2.Schedule Appointment" and "3.Confirm Appointment". However, before progressing to (b), subtasks "1.1.Enter Username" and "1.2.Enter Password" must be completed. In order to progress to the next subtask, tasks 2.1, 2.2 and 2.3 must be carried out, and so on. The dialog model includes different task orders depending on the characteristics of user actions. The action-reaction model of the login task is explained step-by-step, as follows:

1. First, the user identifies herself (task 1).

2. Next, the user completes the next iterative task: "2.Schedule appointment".

3. This task ends when the user confirms the action in subtask 2.3. At this point, the system responds with a predetermined answer or message:

a. In this example, when the user has either too few or too many appointment hours scheduled, the system does not execute the task that usually follows and instead produces a change in interface navigation and in the logic application. A message informs the user of the reason for the change.

b If the correct amount of hours is scheduled, the system executes task "3.Confirm appointments".

This model represents the semantics of the application through interface navigation. When this model is created, verifications of usability and the automatic 
creation of prototypes are possible. This prototype facilitates user and programmer communication. Like Constantine [6], we believe that close coordination between user interface designers and programmers is essential. Therefore, we have incorporated such communication in the requirements analysis.

\section{Conclusions}

InterMod is a methodology based on user-centred models with iterative development cycles and the inclusion of phases for early integration of the user interface in the development process. Additionally, the analysis process permits and facilitates the division of the project into tasks. Using a requirements capture process such as InterMod allows the development process of interactive applications to be agile and reliable.

The requirements models in InterMod include a user model, a system model, a task model and a dialog model. This allows end users and developers to guide, organize and check development.

It is worth noting that this methodology involves developers in navigation design and invites them to participate in dialog model descriptions. This allows for the establishment of critical evaluation points at early stages for the subsequent implementation process. The use of tools that follow the process described in the methodology converts the capture of these requirements into the bridge that connects end users and developers and allows this stage to be used as a guide and a verification of the process.

Acknowledgments. This project has been partially supported by the Spanish Ministry of Education and Science grant TIN2006-14968-C01; and by the University of the Basque Country UPV-EHU, grant EHU06/111.

\section{References}

1. Mayhew, B.: The Usability Engineering Lifecycle. Morgan Kaufmann Publishers, San Francisco (1999)

2. Losada, B., Urretavizcaya, M., Fernández de Castro, I.: The InterMod Methodology: Integrating Software Engineering Processes in Interface Engineering. In: Macías, J.A., Granollers, T., Latorre, P. (eds.). New Trends on Human-Computer Interaction. Springer, Heidelberg (2009)

3. Object Management Group. Model Driven architecture. Technical report (2003)

4. Larman, C.: Agile \& Iterative Development. A manager's Guide. Addison-Wesley/Pearson Education, Boston (2004)

5. Booch, G., Rumbaugh, J., Jacobson, I.: The Unified Modeling Language User Guide. Addison Wesley Longman Inc., Amsterdam (1999)

6. Constantine, L.L., Lockwood, L.A.D.: Usage-Centered Engineering for Web Applications. IEEE Software Magazine 19(1), 42-50 (2002) 